\title{
Des travaux et des cours
}

Industrialisation et enseignement industriel en Europe occidentale des années 1830 aux années 1930

Work and courses. Industrialization and training in Western Europe from the 1830 s to the 1930 s

\section{Stéphane Lembré}

\section{CpenEdition}

Journals

Édition électronique

URL : http://journals.openedition.org/artefact/6882

DOI : 10.4000/artefact.6882

ISSN : 2606-9245

Éditeur :

Association Artefact. Techniques histoire et sciences humaines, Presses universitaires du Midi

Édition imprimée

Date de publication : 7 janvier 2021

Pagination : 335-359

ISBN : 978-2-8107-0706-5

ISSN : 2273-0753

\section{Référence électronique}

Stéphane Lembré, « Des travaux et des cours 》, Artefact [En ligne], 13|2020, mis en ligne le 23

décembre 2020, consulté le 26 décembre 2020. URL : http://journals.openedition.org/artefact/6882 ; DOI : https://doi.org/10.4000/artefact.6882

\section{(c) (i) $\odot$}

Artefact, Techniques, histoire et sciences humaines est mise à disposition selon les termes de la Licence Creative Commons Attribution - Pas d'Utilisation Commerciale - Pas de Modification 4.0 International. 


\section{Des travaux et des cours}

\section{Industrialisation et enseignement industriel en Europe occidentale des années 1830 aux années 1930 \\ Stéphane Lembré}

\section{Résumé}

L'industrialisation de l'Europe qui s'affirme au xixe siècle provoque un vaste bouleversement des qualifications ouvrières. La remise en cause des savoir-faire artisanaux face à la mécanisation ne signifie en rien la disparition des qualifications. La mutation des savoirs et des savoir-faire requis peut être saisie à travers le recours à la formation au travail. Les changements sont cependant complexes selon les métiers, selon les lieux, et sans continuité chronologique parfaite : un vaste ensemble d'institutions, très variées, peut être identifié à travers l'Europe. Leur fondation et leur fonctionnement révèlent la pluralité des motivations de l'enseignement technique industriel, encore cantonné à une minorité des hommes et des femmes jusqu'aux années 1930.

\section{Mots-clés}

apprentissage, formation, histoire, qualification, savoirs, savoir-faire

99 Stéphane Lembré, «Des travaux et des cours. Industrialisation et enseignement industriel en Europe occidentale des années 1830 aux années 1930 », Artefact, 13, 2020, p. 335-359. 


\section{Work and courses. Industrialization and training in Western Europe from the 1830s to the 1930 s}

\section{Abstract}

The industrialisation of Europe, which was asserting itself in the $x x^{\text {th }}$ century, caused a vast upheaval in workers' qualifications. The calling into question of craft skills in the face of mechanisation in no way meant the disappearance of qualifications. The change in the knowledge and skills required can be understood through recourse to on-the-job and vocational training. However, these changes depend on the trades, the location, and without perfect chronological continuity: a vast set of institutions, very varied, can be identified across Europe. Their foundation and operation reveal the plurality of motivations for on-the-job and industrial vocational training, which was still confined to a minority of men and women until the 1930s.

\section{Keywords}

apprenticeship, history, knowledge, know-how, qualification, training 


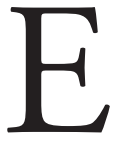
n mettant l'enseignement technique et professionnel et l'apprentissage à l'ordre du jour de la $25^{\mathrm{e}}$ Conférence internationale du Travail, en 1939, le Bureau international du travail (BIT) soulignait l'importance et l'actualité de la question. Vingt ans plus tôt, le préambule de la Constitution de l'Organisation internationale du Travail (dont le BIT est le secrétariat permanent) citait déjà ce domaine au nombre des améliorations à apporter aux conditions de travail ${ }^{1}$.

Il est vrai qu'il n'y avait encore, dans les années 1930, aucune évidence au fait de se former pour exercer un métier, pas plus qu'une définition partagée ne s'était imposée au niveau international sur ce que pouvaient signifier précisément l'apprentissage et l'enseignement technique et professionnel, malgré des efforts en ce sens issus d'un rapport de l'inspecteur général français Paul Jacquemart lors du Congrès international de l'enseignement technique de 1889 à Paris, et comme le confirme la pluralité des termes employés. Pourtant, il est frappant de constater l'émergence de nombreuses institutions de formation destinées aux ouvriers dans les pays d'Europe occidentale engagés dans une phase historique d'industrialisation. Alors que les fondations d'établissements destinés à former des officiers militaires et des ingénieurs avaient débuté plus précocement ${ }^{2}$, la multiplication des cours et des écoles pour les ouvriers s'accélère, sans plan d'ensemble, au cours du XIX ${ }^{\mathrm{e}}$ siècle. Hétérogènes et diffuses, ces institutions érigées dans des contextes nationaux spécifiques ne marquent aucunement la naissance de la formation au travail, pas plus que l'industrie n'apparaît en Europe à cette époque : sauf à présumer l'existence de savoirs et de savoir-faire innés, les métiers ont toujours fait l'objet d'un apprentissage, le plus souvent in situ, c'est-à-dire sur le lieu de travail, par imitation, reproduction et expérience. En revanche, la création d'institutions de formation, substituant à la transmission interpersonnelle sur le lieu de travail, avec ou sans contrat d'apprentissage, des lieux dédiés fréquentés par des groupes d'individus en formation encadrés par des enseignants, ou assurant du moins la coexistence de ces deux modes de formation, constitue une transformation majeure. En dépit de variations nationales dont il faut tenir compte (notamment sur la place de l'apprentissage, qui en est exclue en France avant 1919, mais en est une composante majeure dans l'espace germanique sous la forme des cours du soir donnés dans les Fortbildungschulen),

1. Bureau international du travail, 1939; Lespinet-Moret et Viet, 2011.

2. Voir par exemple : Picon, 1992 ; Morel, 2015. 
l'enseignement technique désigne généralement la structure administrative regroupant l'apprentissage, l'enseignement professionnel et l'enseignement technique supérieur. Dans la logique d'une histoire du travail située à la rencontre entre histoire sociale, histoire économique, histoire politique et histoire culturelle, il s'agit ici de montrer comment, en longue durée et à l'échelle de l'Europe occidentale, l'industrialisation déplace les qualifications requises et transforme les modes de transmission, en centrant l'analyse sur l'émergence de l'enseignement professionnel, défini comme un ensemble d'institutions, cours ou écoles, dédiées à la formation professionnelle d'ouvriers qualifiés, que ce soit au moment de leur entrée en activité professionnelle ou pour le perfectionnement au cours de la carrière. Malgré l'intérêt des enseignements agricoles et commerciaux, on centrera ici l'analyse, dans les limites imparties à un article de synthèse bibliographique, sur la préparation aux métiers de l'industrie.

L'un des risques de la démarche réside toutefois dans la mise en exergue d'un déterminisme qui ferait de l'industrialisation - ou du moins de l'une de ses facettes - la cause unique et nécessaire de l'essor de l'enseignement professionnel. L'argument souvent brandi par les milieux patronaux des "besoins " de formation, qui expliqueraient seuls les caractéristiques de cet essor, masque en effet les choix effectués, le plus souvent par les chefs d'entreprise ou leurs représentants, mais parfois par l'État, des associations voire par les ouvriers eux-mêmes, pour ouvrir, suivre ou au contraire refuser une formation. À défaut de disposer « des matériaux pour une ethnographie comparée des situations d'apprentissage professionnel en France, en Allemagne, en Angleterre et dans les autres pays industriels ${ }^{3}$ ", ainsi que le craignait François Sigaut, un nombre substantiel de travaux, souvent récents, permet la prise en compte des formes et des rythmes divers qu'emprunte l'industrialisation. Au risque de ne pas restituer toute la complexité des évolutions nationales, pour laquelle on renvoie aux travaux cités, l'enjeu consiste à comprendre les modalités du recours à l'enseignement professionnel, qui ne supprime pas toujours ni partout la pratique de l'apprentissage en entreprise, grâce aux comparaisons entre réalisations européennes, entre secteurs d'activité et entre différentes périodes.

3. Sigaut, 1991, p. 35. 


\section{La « crise de l'apprentissage", ou la grande mutation des formations au travail}

S'il est un discours récurrent au cours du XIx ${ }^{e}$ siècle et jusqu'au début du $\mathrm{xx}^{\mathrm{e}}$ siècle, c'est celui de la dégradation de l'apprentissage du métier. Il ne signifie en rien l'abandon complet de l'apprentissage traditionnel, mais en questionne l'efficacité et accentue l'intérêt pour d'autres modalités de formation au travail.

\section{Une institution ancienne et persistante}

Les pratiques en matière d'apprentissage, loin du paradis perdu parfois évoqué aux $\mathrm{XIX}^{\mathrm{e}}$ et $\mathrm{XX}^{\mathrm{e}}$ siècles par les observateurs les plus nostalgiques de l'Ancien Régime ou les plus inquiets de l'intensité de la " crise de l'apprentissage ", étaient variables. La bonne acquisition progressive du métier contrastait avec des situations où l'apprenti était tenu par le maître dans l'ignorance des savoirs et des savoir-faire essentiels, obligeant parfois le premier à profiter des moindres occasions d'apprendre réellement, fût-ce malgré le maître mal intentionné ou mauvais pédagogue, conformément au thème du "métier volé ${ }^{4}$. La révision historiographique opérée à propos des corporations et des guildes, dont la diversité et la souplesse sont désormais mieux connues, se répercute sur l'apprentissage : ainsi, dans le cas de Madrid et à la suite d'autres études de cas, l'étude de plusieurs milliers de contrats d'apprentissage du XVIII ${ }^{\mathrm{e}}$ siècle confirme la nécessité d'envisager les règles qui régissent les corporations comme des références, voire des idéaux, plutôt que comme de fidèles reflets de la réalités. L'apprentissage en tant qu'institution se révèle surtout suffisamment flexible pour permettre que les savoirs et savoir-faire acquis par les apprentis et apprenties soient diversement utilisés par ceux-ci durant leur vie professionnelle. L'institution ne disparaît donc pas brutalement : à Toulouse, en 1889, des apprentis et apprenties seraient employés dans un tiers des entreprises, voire plus des trois quarts pour des métiers comme les tailleurs de pierre,

4. Steffens, 2001.

5. Nieto Sanchez et Zofio Llorente, 2016 ; plus généralement, sur les trajectoires des apprentis d'Amsterdam, Lyon, Leyde et Shrewsbury durant la période moderne, voir Schalk, Wallis, Crowston et Lemercier, 2017. 
carrossiers, fleuristes, forgerons en voitures, pâtissiers ${ }^{6}$. Les caractéristiques de l'apprentissage aux XVIII ${ }^{\mathrm{e}}$ et $\mathrm{XIX}^{\mathrm{e}}$ siècles témoignent aussi du contrôle de la formation professionnelle par les métiers, dont les implications étaient loin d'être uniquement techniques. En Angleterre, où débute la révolution industrielle, les transformations de l'apprentissage sur la longue durée, du $\mathrm{XVII}^{\mathrm{e}}$ au $\mathrm{XX}^{\mathrm{e}}$ siècle, reflétaient le passage de qualifications artisanales à la production en série au sein des usines, mais l'apprentissage pouvait être envisagé comme une garantie de stabilité pour les enfants et leur famille ${ }^{7}$. Ayant choisi de conserver ses corporations, la Prusse promulgue en 1845 le premier Code industriel, puis en 1869 la Gewerbeordnung (étendue en 1871 au Reich), qui théorise le « système dual » dont la formule n'existait pas qu'en Prusse, c'est-à-dire la combinaison entre apprentissage sur le lieu de travail et scolarité à temps partiel, dans un cadre libéral. Jusqu'en 1908, plusieurs modifications de la Gewerbeordnung restaurent les formations d'organisations artisanales de branche puis créent en 1898 les chambres d'artisanat, et consolident une tradition professionnelle face à l'expansion de la production industrielle de masse. Les organisations corporatives urbaines contrôlent ainsi la formation et canalisent les apprentis, secondant les pouvoirs publics inquiets de masses incontrôlées travaillant dans les grandes exploitations industrielles, même si les travailleurs formés partent ensuite prendre des emplois dans l'industrie ${ }^{8}$.

\section{La hantise du déclin}

La dégradation de l'apprentissage au XIX ${ }^{\mathrm{e}}$ siècle est largement dénoncée, dans le sillage de cris d'alarme sur le déclin du savoir-faire ouvrier comme celui du comte de Laborde, en France, dans son rapport rédigé au nom de la section française après l'Exposition universelle organisée au Crystal Palace de Londres en $1851^{9}$. L'inquiétude n'était pas limitée aux industries d'art. L'équipement en machines et l'organisation du travail dans des manufactures menaçaient l'autonomie des métiers, et faisaient craindre une déqualification ouvrière massive faute de suivre le processus productif dans sa globalité ${ }^{10}$. Il s'agissait davantage, en réalité, d'un déplacement des

6. Cité par Lemercier, 2019, p. 100.

7. Lane, 1996 ; Snell, 1996.

8. Voir notamment Greinert, 1995.

9. Laborde, 1856.

10. Jarrige, 2009. 
qualifications, tant l'accroissement du nombre de machines - un objet mal connu et en cours de théorisation - supposait par exemple des mécaniciens capables de les entretenir et de les réparer. Les conséquences sociales et politiques n'en étaient pas moins importantes : elles se mesuraient par la dépossession de l'outil de production, l'équipement en machines supposant un investissement financier hors de portée de la quasi-totalité des ouvriers et ouvrières. L'apprentissage du métier était fortement ébranlé. La législation allemande de la fin du $\mathrm{XIx}^{\mathrm{e}}$ siècle, paradoxale en apparence puisqu'elle restaurait la tradition artisanale alors que le pays connaissait son démarrage industriel, mais compréhensible si l'on rappelle que l'artisanat était bel et bien le principal pourvoyeur de main-d'œuvre à l'échelle de l'empire et que la législation n'empêchait pas les initiatives des milieux industriels, confirme bien la redéfinition de la place de l'apprentissage qui s'impose aux différents États. Les expositions universelles jouent un rôle non négligeable en confrontant les savoir-faire des ouvriers de différents pays. L'idée d'un déclin du savoir-faire et de la menace qu'il faisait courir à la puissance commerciale britannique est ainsi renforcée par l'expérience répétée au fil des expositions, durant la deuxième moitié du XIX ${ }^{\mathrm{e}}$ siècle, d'une infériorité des productions britanniques par rapport à la puissance industrielle et commerciale allemande, et débouche sur des appels au développement de l'enseignement technique ${ }^{11}$.

La prudence s'impose cependant face au diagnostic de "crise de l'apprentissage ", compte tenu d'une part de l'hétérogénéité de l'apprentissage selon les métiers considérés, et d'autre part de l'absence de rupture avec l'Ancien Régime. Dans le cas de Paris, l'étude des contrats d'apprentissage démontre plutôt une grande continuité, qui n'est pas exempte d'actualisations, notamment techniques ${ }^{12}$.

\section{L'enseignement professionnel au secours de l'apprentissage}

Justifiée par les faits ou non, la perception du risque de déclin, particulièrement vive en Grande-Bretagne mais repérable aussi en France, motiva d'abord, côté britannique, après le mouvement des Mechanics' Institutes

11. Edwards, 2008.

12. Crowston, Kaplan et Lemercier, 2018 ; Lemercier, 2019. 
fondés au début des années 1820 et signes d'une première perception de cet enjeu, la fondation en $1878 \mathrm{du}$ City and Guilds of London Institute, destiné à promouvoir l'enseignement technique des garçons et des filles à Londres et dans sa région ${ }^{13}$. Puis, en 1882, l'industriel et député Bernhard Samuelson conclut d'une longue enquête sur l'éducation, la science et l'industrie, que "l'Anglais n'a pas encore appris qu'une éducation étendue et systématique est désormais un préalable nécessaire au développement le plus complet de l'industrie ${ }^{14}$ ", suggérant ainsi les résultats insuffisants des initiatives antérieures. Sur un plan technique et dans un tout autre contexte marqué par la persévérance des structures artisanales, la transformation des compétences requises par l'essor de l'industrialisation se répercute dans le développement d'un enseignement professionnel : à Florence, en Toscane, la Scuola di intagliatori in legno ebanisti e legnajuoli (École de sculpteurs sur bois, ébénistes et menuisiers), créée en 1869, devient une décennie plus tard Scuola professionale di arti decorative e industriali (École professionnelle des arts décoratifs et industriels), et cherche la voie d'une formation artisanale, les transformations successives marquant autant d'ajustements aux changements de l'organisation du travail. Les écoles sont complétées, dans l'offre locale de formation, par " la prolifération d'initiatives plus éphémères : cours du soir, cours professionnels promus par des institutions locales, des groupements professionnels ou des sociétés de secours mutuels, conférences, cycles de leçons de l'Université populaire, etc. ${ }^{15}$ "Un foisonnement comparable s'observe à différents moments au XIX ${ }^{\mathrm{e}}$ siècle : en France dès les années 1820 et 1830, y compris dans des départements ruraux a priori à l'écart de l'industrialisation, et en particulier pour l'enseignement du dessin linéaire. Ce dernier est en principe destiné au peuple, dans la logique d'un enseignement limité aux choses utiles et pratiques ${ }^{16}$.

Les difficultés pratiques (coût, disponibilité d'un enseignant compétent), l'incertitude sur les besoins des ouvriers et la forme pertinente pour transmettre des savoirs et savoir-faire professionnels, ainsi que les réticences à l'égard de formations organisées hors du temps de travail ou difficile d'accès - ne serait-ce que par la langue, pour les ouvriers gallois qui comprennent mal l'anglais, phénomène que l'on retrouve ailleurs, par exemple en Alsace

13. Walker, 2017 ; Gay, 2000.

14. Cité par Sanderson, 1999, p. 1.

15. Pellegrino, 2012.

16. Bodé, 2014 ; sur l'enseignement du dessin : d'Enfert, 2003. 
avant 1870 - se traduisent par des aléas institutionnels dont l'École d'art (School of art) de Swansea constitue un bon exemple : ouverte en 1853 avec 148 élèves, elle ne parvient pas à conserver son public, et rouvre en 1868 sous le nom d'École de science et d'art (School of science and art), à destination d'artisans, d'enfants de commerçants et de la classe moyenne. L'essor de cet établissement date surtout de la fin du siècle, alors que des classes de dessin, qui proposent un enseignement moins ambitieux, attirent plus facilement des élèves ${ }^{17}$.

Dès le milieu du $\mathrm{XIX}^{\mathrm{e}}$ siècle, les évolutions de l'organisation du travail questionnent les modalités de l'apprentissage. En France, après l'enquête de 1863-1964 sur l'enseignement professionnel, qui fournit de nombreuses informations sur la situation de l'apprentissage, l'état des lieux de l'apprentissage effectué sous l'impulsion de l'Office du travail dans les premières années $\mathrm{du} \mathrm{xx}^{\mathrm{e}}$ siècle, qui revient sur les décennies antérieures, aboutit, à partir des cas de l'imprimerie (1901), de l'ameublement (1905) et de l'horlogerie (1911), à la mise en évidence de lacunes graves pour certains métiers ${ }^{18}$. Les responsabilités sont discutées : le progrès technique est invoqué, mais aussi la réglementation sur le travail des enfants ou le temps de travail, et surtout les comportements des familles ouvrières et des patrons, désireux les unes que le travail des adolescents soit rapidement rémunérateur, les seconds de recourir à une main-d'œuvre à moindre coût. Les partisans de l'enseignement technique en école s'appuient sur ces constats pour développer leurs arguments et encourager l'adoption d'une loi sur l'enseignement technique. Les effectifs des apprentis sont certes en baisse, mais sans véritable effondrement, l'absence de contrat écrit en vertu de la loi du 22 février 1851 qui laissait la liberté d'un contrat soit écrit, soit oral, rendant toutefois difficile l'évaluation de la situation. Dans les ateliers d'apprentissage belges, malgré le consensus autour de leur utilité pour les jeunes gens et la création, à la fin du $\mathrm{XIX}^{\mathrm{e}}$ siècle, de nouveaux ateliers centrés sur la pierre et le bois, en plus de ceux spécialisés dans le tissage, le public est très restreint : à la veille de la Première Guerre mondiale, il se limite à 1511 garçons, et dans l'entre-deux-guerres la progression demeure faible ${ }^{19}$.

17. Thomas, 1980 , p. $82-83$.

18. Lespinet-Moret, 2007, p. 159.

19. Grootaers, 1998, p. 371. 
Les nuances indispensables à la description de la dégradation de l'apprentissage n'empêchent pas les progrès, tout au long de la période, d'un enseignement professionnel diversifié qui confirme l'hétérogénéité des formations destinées aux ouvriers. La formation technique relève ainsi, avec l'éducation, des moyens que les élites économiques et politiques peuvent manier pour discipliner la classe ouvrière.

\section{Plasticités des formations ouvrières}

Les normes scolaires actuelles de l'enseignement professionnel, aligné sur la forme scolaire classique, empêchent en partie d'apercevoir les capacités d'adaptation des formations ouvrières dans une époque de faible institutionnalisation. Par leur abondance, leurs finalités et le contrôle dont elles sont l'objet, les formations ouvrières ne peuvent être vues uniquement comme des réponses aux changements techniques, bien que ceux-ci motivent souvent leur action.

\section{L'abondance de l'offre de formation}

Il est difficile de prendre la mesure de l'éventail de formations disponibles, très hétérogènes, plus nombreuses à la ville qu'à la campagne - dont elles ne sont cependant pas absentes -, ainsi que de connaître les élèves qui les fréquentent ou les savoirs enseignés - les brochures ou manuels indiquant davantage ce que l'on se propose d'enseigner que ce qui l'est réellement et ce qui est appris.

À travers l'Europe, les formes que revêtent l'apprentissage (sur le tas) et l'enseignement professionnel (incluant une part scolaire dans la formation) sont très dissemblables. Dans le cas de l'apprentissage, l'existence d'un contrat d'apprentissage permet certes de disposer d'informations précieuses sur les apprentis, filles et garçons, leurs maîtres et les modalités de la formation, mais ne doit pas masquer la part non contractualisée, que d'autres sources, comme les litiges portés devant les conseils de prud'hommes, en France, permettent de dévoiler partiellement. L'enseignement professionnel s'étend des cours saisonniers, éventuellement ambulants à l'image de ces camions-écoles d'artisanat rural qui sillonnent les campagnes françaises dans les années 1920, jusqu'au développement d'établissements constitués en véritables centres de ressources techniques, hébergeant à la 
fois école primaire, école technique de jour et cours du soir et dont les ateliers sont parfois financés en partie par des productions commercialisées. Cette diversité amène à distinguer aussi bien les objectifs assignés à ces formations que la manière dont les ouvriers et ouvrières formés peuvent mobiliser leurs savoirs et savoir-faire dans l'exercice de leur métier.

Malgrél'ampleur des initiatives, au seuil du Xx $x^{\mathrm{e}}$ siècle, le manque d'apprentis est à l'ordre du jour. Les écoles accueillent une faible proportion des jeunes et les cours pour ouvriers adultes n'atteignent qu'une minorité d'entre eux et d'entre elles. La guerre de 1914-1918 a pour effet de relancer, pendant le conflit et durant l'après-guerre, l'attention portée à l'apprentissage et à l'enseignement professionnel : elle place les sociétés industrialisées face au redoutable défi de la conversion puis de la reconversion de l'économie de guerre, les pertes humaines considérables et les « classes creuses » obligeant à se soucier de la productivité. La rationalisation, entendue comme une nouvelle organisation destinée à adapter le processus de production aux mutations nées de la deuxième phase d'industrialisation ouverte depuis les années 1880, a des effets dans des industries modernes comme l'automobile, où certaines entreprises possèdent leurs propres écoles d'apprentissage afin d'ajuster la formation dispensée au plus près de leurs besoins, eux-mêmes redéfinis avec la mise en œuvre dans les années 1920 de l'organisation scientifique du travail ${ }^{20}$. Que des entreprises disposent de leurs propres écoles n'était pas nouveau, mais ce choix, à une époque où l'offre de formation institutionnalisée s'est nettement développée, traduit probablement une méfiance à l'égard des pouvoirs publics et un refus de cursus trop généraux pour former des ouvriers polyvalents alors que l'industrie souhaite des ouvriers adaptés à leur futur poste de travail.

\section{Les finalités}

L'enjeu économique de la formation des ouvriers est assurément central dans l'institutionnalisation de l'enseignement professionnel, dès les années 1830. Dans la toute jeune Belgique, l'Association nationale pour le progrès de l'industrie linière est créée en 1838 afin d'enrayer la crise de l'industrie du lin et du tissage à domicile, née en partie des importations venues d'Angleterre de tissus bon marché, produits avec des procédés mécaniques

20. Hatzfeld, 1989 ; Clarke, 2005. 
et non plus artisanaux. Sous l'impulsion de l'Association, le gouvernement nomme en 1848 une Commission d'enquête sur l'industrie linière qui préconise, parmi différentes mesures, " d'instituer des écoles d'apprentissage de métiers et de faire contribuer aux dépenses les Communes, les bureaux de bienfaisance, autant que possible, et les fonds provinciaux ${ }^{21}$ ".

Cantonner l'enseignement professionnel à la seule transmission de savoirfaire et aux enjeux économiques reviendrait toutefois à méconnaitre les finalités qui lui sont assignées par la plupart de ses promoteurs : il s'agit, selon la formule en vigueur en France à la fin du XIX ${ }^{e}$ siècle et jusquaux années 1930, de former tout à la fois l'homme, le producteur et le citoyen. À l'orée de l'ère industrielle, et pour s'en tenir au cas français, les cours au Conservatoire des arts et métiers à partir de 1819, les cours d'adultes dans les années 1830, les enseignements dispensés par les Associations polytechnique et philotechnique à Paris ou par l'Association philomathique à Bordeaux construisent, en actes, l'enseignement professionnel, en conciliant instruction et formation ${ }^{22}$. La moralisation est une dimension incontournable pour les élites politiques et économiques confrontées aux premiers effets sociaux de l'industrialisation, au moins dans les discours officiels. Elle est particulièrement perceptible dans les formations destinées aux filles, dont la visée professionnelle reste souvent incertaine mais n'est pas exclue en dépit des assignations domestiques produites par les discours officiels, comme l'ont montré Valérie Piette et Éliane Gubin à propos du travail ménager ${ }^{23}$. Pour une activité exercée à domicile et requérant un savoir-faire très long à acquérir comme la dentelle à la main, qui conserve une importance économique au début du $\mathrm{xx}^{\mathrm{e}}$ siècle dans le Puyde-Dôme ou dans le Calvados malgré un déclin engagé en Belgique ou dans le nord de la France, la prévention des effets jugés particulièrement dangereux de la promiscuité entre hommes et femmes à l'usine mobilise encore de nombreux hommes politiques en Belgique comme en France ${ }^{24}$. Inversement, des moments de convergence à l'échelle européenne, à l'instar des années 1860, marquent une intensification des questionnements relatifs à l'enseignement professionnel pour les jeunes filles de la classe moyenne, menacées par un "déclassement » et jugées dignes de faire

21. Neuville, 1976, p. 20.

22. Voir notamment Christen, 2014a et 2014b.

23. Piette et Gubin, 2001.

24. Lembré, 2009. 
l'objet de pratiques philanthropiques ${ }^{25}$. Au total, sur la période considérée, les finalités économiques, techniques, sociales et politiques empêchent toute imputation causale unique à l'essor de l'enseignement industriel.

\section{Contrôler la formation des ouvriers}

Dans la continuité du contrôle par les métiers, l'industrialisation place le patronat en position privilégiée pour assumer le contrôle de l'enseignement industriel, qui reste constamment un enjeu. Le paternalisme en offre une illustration éclatante, puisqu'il inclut le plus souvent la formation professionnelle, dans une visée à la fois technique et éducative. Au Creusot, les Schneider se préoccupent de l'instruction mais aussi de la formation professionnelle des garçons et des filles, les premiers sont destinés aux métiers de la sidérurgie, les secondes aux métiers de bureau ; la même attitude s'observe chez Krupp, où la formation prend place aux côtés du logement parmi les instruments de contrôle de la classe ouvrière ${ }^{26}$.

Dans un pays, l'Italie, où l'industrialisation progresse moins rapidement qu'ailleurs en Europe occidentale, l'enseignement technique constitue une préoccupation des pouvoirs publics, à l'image de l'État naissant : la loi Casati de 1859, importante pour l'ensemble du système éducatif, s'y intéresse au titre du prolongement de l'instruction élémentaire, en tant que formation au travail manuel pour les ouvriers d'industrie, mais de nombreuses réformes dans les années et décennies qui suivent modifient nettement ce qui était alors envisagé, réduisant notamment la part accordée à la pratique en atelier, au profit d'une formation scientifique ${ }^{27}$. En Espagne, l'enseignement technique reste aux mains de quelques grandes entreprises qui l'organisent pour leurs apprentis. Après des initiatives s'inspirant de la France dans les années 1810-1840 sous la forme des escuelas de artes y oficios, les premières écoles publiques sont surtout édifiées à la fin du XIx ${ }^{e}$ siècle et au début du siècle suivant. En 1913, l'Escuela del Trabajo (École du travail) de Barcelone ouvre ses portes à 250 élèves dans une ancienne usine textile en 1913. En dehors des centres urbains industrialisés et notamment de la

25. Albisetti, 2012.

26. Fontaine, 2010 ; James, 2012.

27. Martinelli, 2018. 
Catalogne, la dimension charitable reste prégnante et assimile la formation professionnelle à une éducation destinée aux classes populaires ${ }^{28}$.

La volonté des patrons de conserver le contrôle de la formation professionnelle de leurs salariés les pousse à privilégier l'atelier comme lieu où peut s'effectuer cette formation, ou du moins à n'admettre qu'avec réticence dans le premier tiers du $\mathrm{Xx}^{\mathrm{e}}$ siècle l'essor d'institutions scolaires plus difficiles à gérer : les compagnies minières s'efforcent ainsi de gérer leurs propres institutions de formation et de les réserver à un petit nombre de mineurs, ce qui a pu constituer un frein à la modernisation du travail au fond - l'électrification de l'exploitation ne se généralise ainsi qu'après 1945. C'est tout l'enjeu de la longue discussion de la loi du 25 juillet 1919, dite loi Astier, que de mettre de l'ordre dans les nombreuses institutions d'enseignement technique et professionnel existantes en France, et d'affirmer la place de l'État : le titre V de la loi, le plus innovant, impose l'obligation pour les jeunes garçons et filles de moins de 18 ans employés dans le commerce et dans l'industrie de suivre des cours professionnels, qui peuvent leur permettre de se présenter au certificat d'aptitude professionnelle, diplôme national qui reconnaît leur qualification. De fait, on constate que les législations nationales s'étoffent en ce qui concerne l'enseignement professionnel à la fin du XIX ${ }^{e}$ et au début du $\mathrm{XX}^{\mathrm{e}}$ siècle, dans le sillage de l'affirmation des institutions du travail : la loi Cocco-Ortu du 14 juillet 1907 en Italie, du nom du ministre de l'Agriculture, de l'Industrie et du Commerce de l'époque, classe les écoles professionnelles en cinq groupes et tente d'établir la place de l'État comme devait le faire la loi Astier douze ans plus tard.

L'enjeu du pouvoir sur cet enseignement est aussi révélé par les revendications de certains mouvements féministes, dès la période victorienne, guère couronnées de succès dans la mesure où les formations féminines restent prisonnières de représentations associant les femmes à des métiers "féminins ", rarement industriels alors que les femmes y forment une maind'œuvre loin d'être négligeable, quand les femmes ne sont pas purement et simplement privées de la possibilité d'acquérir des qualifications ${ }^{29}$.

Le contrôle politique est davantage perceptible avec la montée de régimes autoritaires. Leurs dirigeants s'intéressent beaucoup à l'enseignement 
technique, autant sous l'angle de la moralisation de la classe populaire que dans le cadre de conceptions corporatistes et dirigistes de l'économie. Sous la dictature de Primo de Rivera en Espagne (de 1923 à 1930), le Décret royal du 31 octobre 1924 (Statut de l'enseignement industriel) et le Décret royal du 21 décembre 1928 (Statut de la formation professionnelle) cherchaient à " endoctriner les ouvriers ", afin de mener un projet de modernisation économique compatible avec le conservatisme social ${ }^{30}$. Le Troisième Reich se penche à son tour sur l'apprentissage et construit un programme d'enseignement professionnel qui combine formation pratique et endoctrinement politique. En 1938, une loi achève le processus de création d'un programme national intensif de formation au travail en obligeant tous les jeunes qui quittent l'école à suivre une formation industrielle. Bien que les résultats n'atteignent pas les objectifs escomptés, la formation professionnelle est une composante indiscutable de la politique nazie en direction du travail ${ }^{31}$.

\section{Lieux de formation et pédagogies}

Aux formes et aux finalités diverses qu'empruntent les formations au travail correspondent des lieux et des pédagogies qui se renouvellent. La convergence européenne qui s'observe confirme la recherche de solutions pertinentes pour maîtriser les conséquences de l'industrialisation sur les savoirs et les savoir-faire requis pour la main-d'œuvre qualifiée.

\section{Des centres de ressources pour les ouvriers}

La progression du nombre de machines utilisées dans l'industrie et l'affirmation de la science des machines entraînent l'éclosion d'institutions capables de fournir des mécaniciens qualifiés. Si le phénomène est plus précoce en Angleterre compte tenu de la précocité de la révolution industrielle, à Lille, ville industrielle diversifiée malgré la part prépondérante qu'occupe le textile dans l'agglomération qu'elle forme avec Roubaix et Tourcoing, la première machine à vapeur a été introduite en 1818 . Le cours municipal pour chauffeurs mécaniciens s'adresse aux ouvriers adultes à partir de 1858. Il n'est que l'une des composantes de l'offre locale de formation technique,

30. Rico Gomez, 2014.

31. Gillingham, 1986. 
mais ses origines sont intéressantes : sa création intervient deux ans après l'explosion d'une des deux machines à vapeur d'une filature, à Lille, en novembre 1856, qui tue cinq ouvriers et dont la responsabilité est imputée au chauffeur-mécanicien qui avait oublié de remplir la chaudière et au propriétaire de la filature, qui n'avait pas procédé au remplacement du sifflet d'alarme hors d'usage. Le recours à la formation ne naît pas de l'introduction des machines, mais de la preuve de leur dangerosité pour l'homme, tout en confirmant que celui-ci n'est pas tant remplacé par la machine que déplacé par elle. Les chauffeurs-mécaniciens lillois sont alors une véritable aristocratie ouvrière, leur qualification étant reconnue par des salaires nettement supérieurs à ceux du prolétariat ouvrier lillois dont Victor Hugo venait de dénoncer la misère. Face aux accidents fréquents et aux nombreux blessés, la formation devient d'autant plus nécessaire que vers 1862, 629 machines dont 384 pour la seule industrie textile fonctionnent dans l'arrondissement de Lille-Roubaix-Tourcoing. Lécole gratuite des chauffeurs ouvre en janvier 1858, sans véritablement bénéficier du soutien du patronat local, se propose deux objectifs : l'enseignement pratique et la moralisation de la classe ouvrière. Elle fonctionne pendant vingt semaines le vendredi soir avec des leçons publiques et le dimanche matin avec des interrogations pour les auditeurs élèves. L'école ne connaît qu'un succès éphémère - les élèves réguliers, au nombre de 150 en 1858, ne sont plus que 50 en 1861 et 20 deux ans plus tard -, en raison de l'implication qu'elle réclame de la part des ouvriers, de l'absence de retombées professionnelles (notamment salariales) directes, et de la faible reconnaissance par le patronat de l'utilité des cours professionnels d'adultes. Les cours sont cependant relancés dès 1864 par un ancien élève, qui substitue à l'organisation par la Société des sciences locale, une gestion par les ouvriers eux-mêmes, relayés ensuite par la municipalité ${ }^{32}$. Le nord de la France offre comme d'autres territoires industrialisés marqués par la poussée socialiste différentes illustrations de ces expériences d'éducation ouvrière : un autre cours, éphémère et créé par le filateur Charles Junker, mais fondé sur l'auto-éducation des ouvriers, fonctionne ainsi à Roubaix en 1869 avant de s'éteindre progressivement sous la pression des autorités ${ }^{33}$.

La place des machines dans les institutions d'enseignement professionnel est très inégale et reflète la diversité des finalités et des moyens. Ainsi, 
l'apprentissage du dessin constitue une formation professionnelle de base, commune à de nombreux métiers recourant au bois, au fer, à la laine ou au coton. Ressource utile jusque dans de petites villes, cet enseignement s'implante dans de nombreux lieux, sans exiger un matériel onéreux, ce qui écarte l'idée d'une initiation à l'usage des machines, faute, bien souvent, de disposer d'ateliers. Le royaume prussien développe dès la fin du $\mathrm{XVII}^{\mathrm{e}}$ et le début du XVIII ${ }^{\mathrm{e}}$ siècle un réseau d'écoles de perfectionnement (Fortbildungsschulen) destinées aux adultes pour compléter l'instruction primaire, et qui proposent pour une partie d'entre elles (Gewerbliche Fortbildungsschulen) des notions d'instruction pratique autour du dessin, de la géométrie et des mathématiques appliquées ${ }^{34}$. En Italie, l'école de dessin prend aussi une importance centrale au tournant du XvIII ${ }^{\mathrm{e}}$ et du $\mathrm{XIX}^{\mathrm{e}}$ siècle ; cet enseignement est pris en charge par diverses institutions, par exemple des sociétés d'entraide dans le Piémont qui en perçoivent le caractère incontournable pour prétendre s'élever au-dessus de la maind'œuvre non qualifiée ${ }^{35}$.

Parmi les centres de ressources ouverts aux ouvriers et aux ouvrières, certains restent sous le contrôle direct ou indirect de ceux-ci. Les Bourses du travail en fournissent un très bon exemple au seuil du $\mathrm{xIx}^{\mathrm{e}}$ siècle, malgré la brièveté de l'expérience dans la plupart des cas, de même que l'Université du travail de Charleroi, qui naît d'écoles professionnelles initialement créées en 1903 par les industriels du Hainaut ${ }^{36}$. Dans cette université qui s'inspire de réalisations étrangères, notamment états-uniennes, avant d'être citée comme une référence en Europe dans les années 1920, sont regroupés plusieurs cours de différents niveaux et concernant plusieurs métiers : la part de la formation des ouvrières et des ouvriers et de celle d'un personnel d'encadrement reste à mesurer en étudiant les parcours professionnels. Pour l'année scolaire 1923-1924, 2183 élèves fréquentent les cours permanents, 204 les cours temporaires, et l'université délivre 478 diplômes. Le succès de cette institution qui fournit un enseignement professionnel reconnu par les entreprises de la région oblige la direction à introduire des examens d'admission ${ }^{37}$.

34. Harney, 1980.

35. Allio, Gera, 1992.

36. Buyse, 1914.

37. Informations sociales, 30 mars 1925. 


\section{Mobilités ouvrières de formation}

Bien qu'elles soient mieux connues pour la formation des ingénieurs, les mobilités ouvrières sont intenses au XIX ${ }^{\mathrm{e}}$ siècle et pas seulement guidées par la misère. Elles sont régulièrement motivées par la formation et prenaient alors la forme d'un " tour ", comme le révèle le cas du dessinateur textile alsacien Henri Lebert (1794-1862), auteur d'un journal où il consigne l'ensemble de ce qu'il apprend lors de ses déplacements, tout au long de sa carrière $^{38}$. Dans les régions allemandes de la première moitié du siècle, plusieurs années de voyage étaient légalement requises pour pouvoir prétendre à la maîtrise et au statut d'artisan. Au milieu du siècle, des ouvriers qualifiés belges viennent parfaire leur apprentissage d'ébéniste ou de cordonnier à Paris avant de s'établir en Belgique. Les déplacements étaient souvent de moindre ampleur, à l'image de ce maréchal-ferrant né dans la petite ville de Diest, dans le Brabant flamand, qui accomplit son apprentissage dans le village d'Olmen, puis travaille dans d'autres ateliers ruraux, avant de revenir s'établir à Diest vers 1900. Le phénomène n'est pas massif et il reste peu structuré, mais Sven Steffens a montré qu'il concernait un nombre substantiel d'ouvriers qualifiés ruraux ou urbains ${ }^{39}$.

Ces migrations qualifiantes font de la succession des expériences de travail une accumulation de savoirs et de savoir-faire. L'institution du compagnonnage y ajoute la force des rites et la dimension initiatique, comme le relate Agricol Perdiguier, un ouvrier menuisier qui raconte a posteriori son tour de France ${ }^{40}$, et dont s'inspire George Sand, opposant de manière romancée le savoir-faire d'un fils acquis grâce à son " tour " aux préventions du père, ébéniste comme lui mais initialement plein de dédain à l'égard de l'institution compagnonnique ${ }^{41}$.

\section{Les travailleurs, l'exposition et le musée}

Un autre lieu de formation, fondé également sur le déplacement, concerne les expositions. Partie émergée de la frénésie d'expositions et de concours à tous niveaux que connaissent les pays en cours d'industrialisation

38. Millet, 2018.

39. Steffens, 2003.

40. Perdiguier, 2002.

41. Sand, 1869. 
jusqu'en 1914, les événements-mondes que constituent les expositions universelles à la suite de celle de Londres, en 1851, attirent de nombreux ouvriers, parfois envoyés en délégations par des gouvernements ou des organisations syndicales. S'ils y découvrent de nombreuses machines nouvelles, ériger ces expositions en moment de la perception par les ouvriers de leur dépossession du travail cantonne ces derniers à un rôle de spectateurs passifs du progrès technique qui ne rend pas raison de ce qui se joue alors ${ }^{42}$. Ainsi, la présence des ouvriers italiens aux expositions, renforcée à partir de l'unification italienne et de l'exposition de Vienne, en 1873, s'accompagne d'un rapport ambivalent au progrès technique. Bien que les élites économiques italiennes comptent sur les expositions pour s'approprier les savoir-faire de grandes puissances industrialisées et moderniser l'appareil de production italien, les travailleurs qualifiés décrient le progrès technique plutôt qu'ils ne l'admirent. La visite à l'exposition n'en participe pas moins à une acculturation au progrès technique qui contribue à la formation technique de ces spectateurs avertis ${ }^{43}$.

Les musées industriels jouent un rôle également important. En France, le Conservatoire des arts et métiers fait office de rôle pionnier et de nombreuses écoles, publiques comme privées, comprenaient un musée industriel. La présence de cet espace dans les écoles se répand, y compris dans des régions faiblement industrialisées : en 1880, à Naples, le projet d'organisation combinant musée d'art industriel et écoles-ateliers de Naples, fondé sur une conception artisanale du savoir-faire et porté par un groupe d'intellectuels réunis par un notable, Gaetano Filangieri junior, reprenait le modèle du South Kensington Museum constitué en 185244. Les expositions universelles et les musées sont la partie la plus visible d'une pédagogie par l'observation et la démonstration, dont le rôle aux côtés de l'apprentissage par la pratique est probablement sous-estimé. Nombre d'écoles industrielles possèdent leurs propres musées. C'est le cas à Naples, mais aussi, par exemple, à l'École nationale des arts industriels de Roubaix qui enrichit soigneusement des collections d'étoffes observées par les ouvriers qualifiés du textile, dont les employeurs demandent qu'ils puissent accéder au musée installé au cour de l'établissement y compris sur leur temps

42. Rancière et Vauday, 1975, p. 6.

43. Pellegrino, 2017

44. Santillo, 2015 ; Argles, 1964. 
libre ${ }^{45}$. À Florence, la Bibliothèque d'art industriel, rebaptisée par la suite Bibliothèque populaire industrielle, suggère l'importance des engagements locaux en faveur de la formation technique des ouvriers ${ }^{46}$. Les cas italiens s'inscrivent dans une politique volontariste de développement d'une éducation industrielle et artistique jugée décisive pour intensifier l'industrialisation et "rattraper" les concurrents européens ${ }^{47}$.

L'ensemble de l'enseignement industriel, qu'il soit destiné à des filles et des garçons déjà entrés dans le monde du travail ou sur le point d'achever leur formation, concerne toujours une minorité jusqu'à la Deuxième Guerre mondiale. Pourtant, sans accroissement brutal des effectifs, dans le sillage de la crise économique, les années 1930 engagent en Europe occidentale une nouvelle période pour la formation au travail. Alors que les enjeux économiques et techniques de préparation au travail restent déterminants, avec l'ampleur inédite que prend le chômage, la formation est aussi chargée de la rééducation professionnelle, comme c'était le cas depuis la loi Astier et dans les Fortbildungschulen germaniques. Cette dernière fonction passe préférentiellement par des centres et des écoles qui renvoient au mouvement de longue durée de relégation de l'apprentissage à l'arrière-plan des modes de formation professionnelle, l'enseignement professionnel en école étant jugé plus efficace pour doter les travailleurs de qualifications tandis qu'au moins en France voire en Grande-Bretagne, l'apprentissage change de forme, sinon de signification.

La comparaison des projets et des réalisations dans divers pays d'Europe occidentale durant un siècle d'industrialisation, sans prétention aucune à l'exhaustivité, permet néanmoins de rappeler la multiplicité des formes que revêt l'enseignement industriel et de démontrer, par-delà les dissemblances, la pluralité des facteurs de transformation des modes de formation. Sans sous-estimer les spécificités des évolutions nationales qui mériteraient, de même que les différences dans l'offre de formation pour les mains-d'œuvre selon les sexes et selon les nationalités, d'être présentées plus en détail, cet article pose les jalons d'une déconstruction de modèles nationaux parfois décrits sans attention aux cheminements historiques

45. Lembré, 2017.

46. Pellegrino, 2017, p. 143.

47. Martinelli, 2018. 
relativement similaires à l'échelle européenne ${ }^{48}$. La démonstration est également nécessaire, intégrant les nombreux discours sur la prise en compte des évolutions techniques dans la préparation aux transformations des mondes du travail, non pour nier la réalité de causes techniques à la remise en cause de l'apprentissage " traditionnel ", érigeant le lieu de travail en espace évident de l'apprentissage du métier, mais pour mieux apprécier la place exacte de ces facteurs techniques et les circonstances dans lesquels ils influencent des décisions de création, de réforme voire d'interruption de dispositifs de formation.

\section{Sources}

Bureau international du Travail, Informations sociales, vol. XIII, $\mathrm{n}^{\circ}$ 13, 30 mars 1925.

Buyse Omer, Une université du travail, $\mathrm{H}$. Dunod et E. Pinat, Paris, 1914.

Conférence internationale du Travail, $25^{e}$ session, Genève 1939. Enseignement technique et professionnel et Apprentissage. Première question à l'ordre du jour. Rapport I, Bureau international du travail, Genève, 1939.

LaBorde Léon de, De l'union des arts et de l'industrie, Imprimerie Impériale, Paris, 1856.

Enquête sur l'enseignement professionnel ou Recueil de dépositions faites en 1863 et 1864 devant la Commission de l'enseignement professionnel, Imprimerie impériale, Paris, 1864-1865, 2 vol.

Perdiguier Agricol, Mémoires d'un compagnon, rééd. La Découverte, Paris, 2002. SAnd Georges, Le Compagnon du tour de France, reéd. Michel Lévy, Paris, 1869.

\section{Bibliographie}

Alberdi Ramon, La formación profesional en Barcelona: política, pensamiento, instituciones, 1875-1923, Don Bosco, Barcelona, 1980.

Albisetti James C., "Philanthropy for the middle class: vocational education for girls and young women in mid-Victorian Europe", History of Education, vol. 41, n 3, 2012, p. 287-301.

Allio Renata, Gera Bianca, "Società di mutuo soccorso e scuole professionali in Piemonte : esperienze passate e presenti ", dans Instruire le peuple. Education populaire et formation professionnelle dans la France du Sud-Est et l'Italie du Nord $X V I I T^{\top}-X X^{e}$ siècles, Centre de recherches d'histoire de l'Italie et des Pays alpins, Grenoble, 1992, p. 81-94.

48. Greinert, 2005. 
Argles Michael, South Kensington to Robbins: An Account of English Technical and Scientific Education since 1851, Longmans, Londres, 1964.

Bodé Gérard, "Les cours professionnels pour ouvriers sous la Restauration et la monarchie de Juillet ", Les Études Sociales, 2014-1, n 159, p. 29-47.

Bodé Gérard, Marchand Philippe (dir.), Formation professionnelle et apprentissage XVII'-XX siècles, Revue du Nord, INRP, Villeneuve-d'Ascq, Paris, 2003.

Christen Carole, "Les cours pour ouvriers adultes au Conservatoire des arts et métiers dans le premier XIX ${ }^{\mathrm{e}}$ siècle ", Cahiers de RECITS, n 10, 2014, p. 33-56.

Christen Carole, "Jalons pour une histoire de l'éducation industrielle destinée aux ouvriers adultes au XIX ${ }^{\mathrm{e}}$ siècle ", Les Études sociales, $\mathrm{n}^{\circ} 159,2014$, p. 3-9

Clarke Constanze, Automotive Production Systems and Standardisation. From Ford to the Case of Mercedes-Benz, Physica-Verlag, Heidelberg, 2005.

Crowston Clare H., Kaplan Steven Laurence, Lemercier Claire, «Les apprentissages parisiens aux XVIII ${ }^{\mathrm{e}}$ et $\mathrm{XIX}^{\mathrm{e}}$ siècles ", Annales. Histoire, Sciences Sociales, $\mathrm{n}^{\circ} 4,2018$, p. 849-889.

D’Amico Nicola, Storia della formazione professionale in Italia. Dall'uomo da lavoro al lavoro per l'uomo, Franco Angeli, Milano, 2015.

D'Enfert Renaud, L'Enseignement du dessin en France. Figure humaine et dessin géométrique (1750-1850), Belin, Paris, 2003.

EDWARds Anthony David, The Role of International Exhibitions in Britain 18501910. Perceptions of Economic Decline and the Technical Education Issue, Cambria Press, Amherst, New York, 2008.

FontaIne Jacqueline, La Scolarisation et la formation professionnelle des filles au pays de Schneider (1844-1942), L'Harmattan, Paris, 2010.

GAY Hannah, "Association and practice: the city and guilds of London Institute for the Advancement of Technical Education", Annals of Science, $n^{\circ}$ 57, 2000, p. 369-398.

Gillingham John, “The 'deproletarianization’ of German society: vocational training in the Third Reich", Journal of Social History, vol. 19, 1986, p. 423-432.

GaYot Gérard, Minard Philippe (dir.), Les Ouvriers qualifiés de l'industrie (XVI$X X^{e}$ siècle). Formation, emploi, migrations, Revue du Nord, hors série n ${ }^{\circ}$ 15, 2001.

Goodman Joyce, "Social investigation and economic empowerment: the Women's Industrial Council and the LCC Trade Schools for Girls, 1892-1914”, History of Education, vol. 27, $\mathrm{n}^{\circ} 3,1998$, p. 297-314.

Greinert Wolf-Dietrich, Das „deutsche System“der Berufsausbildung: Geschichte, Organisation, Perspektiven, Nomos Verlagsgesellschaft, Baden-Baden, 1995.

Greinert Wolf-Dietrich, Mass Vocational Education and Training in Europe: Classical Models of the 19th Century and Training in England, France and Germany during the First Half of the 20th, Office for official publications of the European Communities, Luxembourg, 2005. 
Grootaers Dominique (dir.), Histoire de l'enseignement en Belgique, CRISP, Bruxelles, 1998.

Harney Klaus, Die preußische Fortbildungsschule. Eine Studie zum Problem der Hierarchisierung beruflicher Schultypen im 19. Jahrhundert, Beltz Verlag, Weinheim, Bâle, 1980.

Hatzfeld Nicolas, «L'école d'apprentissage Peugeot (1930-1970). Une formation d'excellence ", Formation Emploi, n' 27-28, 1989, p. 115-128.

James Harold, Krupp: A History of the Legendary German Firm, Princeton University Press, Princeton, 2012.

JARRIGe François, Au temps des tueuses de bras: les bris de machines à l'aube de l'ère industrielle, 1780-1860, Presses universitaires de Rennes, Rennes, 2009.

Lane Joan, Apprenticeship in England: 1600-1914, UCL Press ltd, Londres, 1996.

Lembré Stéphane, "La flamme qui brûle et la flamme qui éclaire" : une expérience d'éducation ouvrière à Roubaix, 1869-1872 ", Les Études Sociales, n 159, 2014, p. 133-148.

Lembré Stéphane, «Les écoles de dentellières en France et en Belgique des années 1850 aux années 1930 ", Histoire de l'éducation, n 123, 2009, p. 45-70.

Lemercier Claire, "À qui l'apprentissage donne-t-il du pouvoir ? (France, $\mathrm{XIX}^{\mathrm{e}}$ siècle) ", Mélanges de l'École française de Rome. Italie et Méditerranée modernes et contemporaines, $\mathrm{n}^{\circ}$ 131-1, 2019, p. 99-113.

Lespinet-Moret Isabelle, L'Office du Travail. La République et la réforme sociale, Presses universitaires de Rennes, Rennes, 2007.

Lespinet-MoREt Isabelle, VIET Vincent (dir.), L'Organisation internationale du travail: origine, développement, avenir, Presses universitaires de Rennes, Rennes, 2011.

MarChand Philippe, "Le Cours municipal des chauffeurs-mécaniciens de Lille, 1858-1939. Une expérience professionnelle d'adultes ", Histoire de l'éducation, n 66,1995 , p. 137-158.

Martinelli Chiara, "Searching for a national model. Early paths in Italian industrial and artistic-industrial education ", Rivista di storia dell'educazione, $\mathrm{n}^{\circ} 2$, 2018, p. 249-267.

Millet Audrey, Vie et destin d'un dessinateur textile : d'après le journal d'Henri Lebert, 1794-1862, Champ Vallon, Seyssel, 2018.

Morel Thomas, Von der akademischen zur praktischen Mathematik (1765-1851). Mathematisch-technische Bildung zwischen sächsischer Bergakademie und polytechnischer Schule Dresden, Diachron-Verlag, Berlin, 2015.

Neuville Jean, La Condition ouvrière au XIX siècle. Tome I, L'ouvrier objet, Vie Ouvrière, Bruxelles, 1976. 
Nieto Sanchez José Antolin, Zofio Llorente Juan Carlos, "The Return of the Guilds: A View from Early Modern Madrid”, Journal of Social History, vol. 50, $\mathrm{n}^{\circ} 2,2016$, p. 247-272.

Pellegrino Anna, La città più artigiana d'Italia. Firenze 1861-1929, Franco Angeli, Milan, 2012.

Pellegrino Anna, Les Fées machines. Les ouvriers italiens aux Expositions universelles (1851-1911), Classiques Garnier, Paris, 2017.

Picon Antoine, L'Invention de l'ingénieur moderne : l'École des Ponts et Chaussées, 1747-1851, Presses de l'École nationale des ponts et chaussées, Paris, 1992.

Piette Valérie, Gubin Éliane, "Travail ou non-travail ? Essai sur le travail ménager dans l'entre-deux-guerres ", Revue belge de philologie et d'histoire, n 79-2, 2001, p. 645-678.

Rancière Jacques, VAuday Patrice, «En allant à l'expo : l'ouvrier, sa femme et les machines ", Les Révoltes logiques, $\mathrm{n}^{\circ}$ 1, 1975, p. 5-22.

Rico Gómez Maria-Luisa, La formación profesional obrera en España durante la dictadura de Primo de Rivera y la Segunda República, Consejo Superior de Investigaciones Cientificas, Madrid, 2014.

SANDERSON Michael, Education and economic decline in Britain, 1870 to the 1990s, Cambridge University Press, Cambridge, 1999.

Santillo Marco, "L'échec du projet de Gaetano Filangieri junior en faveur du développement économique du Mezzogiorno : la courte saison du musée d'Art industriel et des écoles-ateliers de Naples ", dans Coquery Natacha et DE Oliveira Matthieu (dir.), L'Échec a-t-il des vertus économiques ?, Comité pour l'histoire économique et financière de la France, Paris, 2015, p. 297-307.

Schalk Ruben, Wallis Patrick, Crowston Clare, Lemercier Claire, "Failure or Flexibility? Apprenticeship Training in Premodern Europe", Journal of Interdisciplinary History, vol. XVIII, n 2, 2017, p. 131-158.

Sigaut François, "L'apprentissage vu par les ethnologues. Un stéréotype ? ", dans Chevallier Denis (dir.), Savoir faire et pouvoir transmettre, Maison des sciences de l'homme, Paris, 1991.

SNELL Keith D. M., "The apprenticeship system in British history: the fragmentation of a cultural institution", History of Education, $n^{\circ} 25,1996$, p. 303-321.

Steffens Sven, "Le métier volé. Transmission des savoir-faire et socialisation dans les métiers qualifiés au XIx ${ }^{\mathrm{e}}$ siècle (Belgique-Allemagne) ", Revue du Nord, hors-série $\mathrm{n}^{\circ} 15,2001$, p. 121-135.

Steffens Sven, "Migrations ouvrières temporaires de formation professionnelle en Belgique (de l'Ancien Régime à 1914). Première approche et comparaison avec la France et l'Allemagne ", dans Fillieux Véronique, Honnoré Laurent et Servais Paul (éd.), Angles d'approches. Histoire économique et sociale de l'espace 
wallon et de ses marges (XV'-XX siècles), Bruylant-Academia, Louvain-la-Neuve, 2003, p. 159-174.

Thivend Marianne (dir.), "Apprentissages et formations techniques et professionnelles de filles et de garçons, $\mathrm{XIX}^{\mathrm{e}}$ et $\mathrm{Xx}^{\mathrm{e}}$ siècles ", Cahiers Pierre Léon d'histoire économique et sociale, $\mathrm{n}^{\circ}$ 6, 2005.

Thomas J.-P., "A consideration of technical education in the Swansea district, 1850-1900", The Vocational Aspect of Education, vol. xxII, $\mathrm{n}^{\circ}$ 83, december 1980, p. 81-90.

WaLker Martyn, The Development of the Mechanics'Institute Movement in Britain and Beyond. Supporting further education for the adult working classes, Routledge, London, 2017.

\section{Lauteur}

Stéphane Lembré est maître de conférences en histoire contemporaine à l'Université de Lille (INSPE), chercheur au CREHS (Université d'Artois). Ses recherches portent sur la formation au travail et l'éducation aux $x x^{\mathrm{e}}-\mathrm{xx} \mathrm{x}^{\mathrm{e}}$ siècles. Publications récentes : « L'enseignement professionnel et la culture ouvrière sous la Troisième République ", Mil neuf cent. Revue d'histoire intellectuelle, n 35, 2017, p. 41-59; avec Jérôme Krop (dir.), Histoire des élèves en France, vol. 2, Ordres, désordres et engagements (XVI- $x x^{e}$ siècles), Villeneuve-d'Ascq, Presses universitaires du Septentrion, 2020. Contact : stephane.lembre@inspe-lille-hdf.fr 